one or two minutes. The blotching character of the red line noticeable in states of fatigue, as well as following a period of reduced oxygen supply, disappears after the inhalation, and the puckering of the skin and clear edges of the line suggest that a decided improvement in the vascular tone has taken place.

\section{THE OCCURRENCE AND SIGNIFICANCE OF B. WELCHII IN CERTAIN WOUNDS *}

\author{
JAMES L. STODDARD, M.D. (Boston) \\ Captain, M. R. C., U. S. Army \\ FRANCE
}

It is well known that $B$. welchii is usually very common in early wounds. Studies by Fleming ${ }^{1}$ in 19141915 showed its presence in 81 per cent. of 127 wounds from one to seven days old ; in 34 per cent. of fifty-six wounds of from eight to twenty days, and in 18.5 per cent. of wounds more than twenty days old. Goadby ${ }^{2}$ presents figures for the occurrence of anaerobes in wounds, but none for the incidence of $B$. welchii in particular. He found a markedly less percentage of saccharolytic and ensporing anaerobes in excised than in nonexcised wounds. Plisson ${ }^{3}$ found harmless persistence of $B$. welchii until the tenth day in twentytwo of 186 cases, and in three beyond the fifteenth day. Taylor ${ }^{4}$ proved the presence of gas-forming bacilli on sequestrums in 14 per cent. of a series of wounds with sequestrums aged from forty-five to 300 days.

Taylor's figures show that $B$. zelchii can occur and persist for a very long time on bone and in deep parts of wounds. Whether cultures from the exudate would be positive in these cases is a different question. Taylor considers the persistence to depend on the protection of the bacteria from the body fluids; hence it seems improbable that the bacilli would appear in the exudate taken by the usual cultural methods.

Since the observations recorded here were made partly to discover as far as possible the significance of the usual cultural examination of wounds for B. welchii, cultures were taken by the routine methods, which consisted in (1) taking specimens of the exudate on cotton swabs; (2) planting in recently boiled and cooled milk of neutral reaction, and ( 3 ) anaerobiosis by the pyrosoda method of Wright or by the hydrogen and palladium asbestos method. Milk is not quite so good a cultural medium for $B$. weichii as glucose bouillon, for it takes a slightly heavy inoculation to get growth. It is used, however, because it is the only practicable method of getting a characteristic culture in twentyfour hours, and hence is the usual routine test. No culture method gives growth with doses under a certain size; hence the absolute presence or absence cannot be ascertained in any event.

The points especially considered here are: (1) the occurrence of $B$. welchii (as determined by the ustal culture test) in wounds at a base hospital during a quiet period when casualty clearing station treatment

*From U. S. Army Base Hospital No. 5.

1. Fleming, Alexander: The Bacteriology of Septic Wounds, Lancet, London, $1915, \boldsymbol{2}, 638$.

2. Goadby, K.: The Bacterial Flora of War Wounds, Brit. Med. Jour., 1918, 1, 581.

3. Plisson, L.; Ramond, and Vergelet, C.: The Bacterial Flora of War Wounds, Bull. Soc. Méd. d. Hóp. de Paris, Dec. 21, 1917, p. 1302.

4. Taylor, K.: Persistence of Bacteria within Sequestra, Ann. Surg., $1917, \mathbf{6 6}, 522$. is thorough; (2) the occurrence of gas infection in the same series and its relation to the occurrence of $B$. welchii; (3) the persistence of $B$. welchii after gas. infection, compared with its persistence in wounds in which no gas infection has occurred; $(4)$ the value of examination for $B$. welchii in regard to the diagnosis of cases of gas infection occurring in excised wounds, and (5) the relation of $B$. welchii to suture.

\section{THE OCCURRENCE OF B. WELCHII IN WOUNDS AFTER THOROUGH CASUALTY CLEARING STATION TREATMENT}

It seemed probable that a quiet period would afford an opportunity to determine the effectiveness of casualty clearing station treatment, when at its best, in reducing the occurience of $B$. welchii in wounds. During the time of the study, a culture was taken of each wound at the first dressing or soon after, of patients arriving at the hospital. Only wounds involving muscles were included. Trivial wounds and clean bullet wounds were eliminated. A consecutive series of 137 cases was thus obtained.

$B$. welchii was proved present by culture in 23 per cent. of the cases. Of nineteen wounds of one day, $B$. welchii occurred in 26 per cent. Of forty-four wounds of two days, it was found in 20 per cent.; in twenty-seven wounds of three dacys, in 19 per cent.; in thirty-five wounds of from four to seven days, in 25 per cent., and of four wounds of from eight to ten days, in 50 per cent. Eliminating the figures for eight to ten days as based on too few cases, the occurrence is markedly uniform in wounds of the different ages.

The occurrence of the same or a higher incidence in older cases is explicable by the fact that these patients came late to the hospital, largely because they had unusually severe wounds or had had a previous gas infection at the casualty clearing station. In view of the persistence of $B$. welchii after gas infection (described later), the incidence was calculated in wounds that had not had a previous gas infection. The incidence for wounds up to four days remained the same, but for wounds of from four to seven days it fell from 25 to 16 per cent., and for the whole series from 23 to 19 per cent.

Smear examination was done merely to obtain a rough idea of the numbers of $B$. welchii present. As a rule, only two distinctions were made: $(a)$ those having no $B$. welchii or extremely few, so that a considerable search was necessary to find them; these are called negative; $(b)$ those having more; these are called positive.

The incidence of cases with positive smear and culture (eliminating cases with previous gas infections) was: in one day wounds, 18 per cent.; in wounds of two days, 18.6 per cent.; in wounds of three days, 8 . per cent.; in wounds of from four to seven days, 11 per cent., and in the series as a whole, 14.5 per cent.

These low percentages for the occurrence of $B$. welchii seem to indicate extremely effective previous treatment. The influence of excision at first seems to be absent, for of the cases in which excision was performed, according to the field medical card, $B$. zelchii was present in 23.5 per cent., while of those not stated to be excised it was present in 19.4 per cent. The explanation lies in the fact that the more severe wounds are the ones that are excised and are also the ones in which $B$. welchii is most likely to occur. Eliminating cases with previous gas infection, the occur- 
rence of $B$. welchii in the excised wounds is not lower than in the nonexcised. These results suggest, at least, that good judgment was used in performing excision, giving a very uniform output of cases.

Goadby's finding of a higher percentage of saccharolytic anaerobes in nonexcised than in excised wounds has only the appearance of differing from these figures. His nonexcised cases included nearly all cases at a period when excisions were little done, while his excised wounds occurred at a different period when the percentage of excisions was high. Consequently each of his series included wounds of all sorts, while in this series the nonexcised were merely the lighter wounds left over after the severe ones had been done.

INCIDENCE OF GAS INFECTION IN THE SERIES

Definite muscle gas infection after arrival occurred in the series in 2.9 per cent. of the cases. If a few slightly doubtful cases are included, the total is 5.1 per cent. Of the cases with $B$. welchii on culture, unquestionable gas infection occurred in 13 per cent.; including slightly doubtful cases, the figure becomes 22.5 per cent.

THE PERSISTENCE OF B. WELCHII IN THE WOUNDS

The cases in which $B$. zelchii was found were followed, as far as possible, until the cultures became negative. Usually more than one negative examination was obtained. Besides the cases in this series, records are included from a previous series of 200 nonconsecutive cases.

In the cases without gas infection, a fairly marked change from positive to negative was evident by the eightl? day of the wound. Before this, 30 per cent. had become negative; but after this day, only 17 per cent. remained positive, and these for a short time, as a rule.

Of the cases with gas infection, the period of change from positive to negative cultures was not evident until about the twelfth day of the wound; before this, only 12 per cent. had become negative; while afterward, 36 per cent. remained positive.

Thus there is evidently a greater tendency for the iersistence of $B$. welchii in wounds having muscle ii fection. This is in spite of the fact that each case of gas infection had thorough removal of débris and excision, and in each case the symptoms of an active infection stopped immediately after the operation. Even if the reckoning is made from the time of the operation as a starting point, the period of change comes on the tenth or eleventh day. The persistence was remarkable on casual observation before the figures were obtained.

A persistence in noticeable numbers in smears occurred in the gas infection series until about the eighth day of the wound. There seemed to be a fairly regular diminution in numbers until the eighth day, and 43 per cent. had become negative by that time, while 20 per cent. remained positive beyond the eighth day. In the cases without gas infection, more than 75 per cent. had become negative before the eighth day, and only 15 per cent. remained positive beyord it.

These figures indicate the tendency to persisi longer in moderate numbers when a gas infection has preexisted. In fact, in wounds without gas infection the tendency is strong for bacilli to become very few early in the history of the wound. Half of the smears had become negative by the fifth day, while by that time only 18 per cent. had become negative on culture.
Of the gas-infected cases, however, only 12 per cent. of the smears had become negative by the fifth day, and none by culture.

These results suggest that $B$. welchii does not flourish long in surface exudate. The time of persistence in gas infection cases, with the gradual diminution in numbers, suggests either foci of infection, gradually overcome, superficial growth in the exudate, or a gradual exudation of the bacteria from the previously infected tissues. Local foci of active infection seem extremely unlikely in view of the thorough operation in each case, and the entire absence of clinical evidence of infection. A superficial growth in the exudate might account for the early persistence in considerable numbers; but the long, late persistence in numbers practically imperceptible in the smear suggests rather gradual riddance of the bacteria from the infected muscle. Such a process would also account for the large numbers soon after operation. Pathologic studies show the presence of $B$. welchii beyond the area of apparent infection, and it is often easy in cases of heavily infected wounds to demonstrate the passage of bacteria from within the infected wall.

The marked tendency to early dying out in numbers in cases not infected is additional evidence of the difficulty of the continued growth of $B$. welchii in surface exudate in open wounds. There are always a certain number of cases of slight local gas infections which are not noted clinically. It seems probable that this must account for a certain number of the instances of persistence in the cases classified as not infected. This makes the probable true persistence in moderate numbers very small. The later persistence in very small numbers is probably to be explained by the presence of small foci, such as pockets, in which favorable anaerobic conditions are obtained; it is most noticeable in very large wounds which are apparently quite open, but in which, on account of the magnitude of operation, it is difficult to carry out a complete excision or removal of débris.

\section{THE VALUE OF EXAMiNATION FOR B. Welchi}

It is apparent on examination of the slides that in cases of active gas infection, bacilli are usually present in much larger numbers than in wounds of the same age without gas infection. Thus, of the wounds of from one to three days with $B$. welchii on culture but without gas infection, 40 per cent. showed negative smears; 46 per cent showed very moderate numbers, and only 4 per cent. showed fairly large numbers. Of the gas-infected cases (twenty in number), 5 per cent. showed negative smears, 40 per cent. moderate numbers, and 55 per cent. large numbers. Thus the finding of large numbers of $B$. zelchii in well excised wounds of from one to three days seems strong presumptive evidence of active gas infection. (It may be remarked that of these particular gas-infected wounds, practically all had been excised.) The older the wound, the stronger the evidencè.

If the cultures are positive but the smears negative. or if the cultures are negative, it is somewhat strong evidence against gas infection. It is not at all conclusive evidence, for in certain cases of gas infection, $B$. welchii may be absent altogether. In other cases, in which $B$. welchii is a factor, the infection may begin about a foreign body deep in the wound and the culture be negative. It does not seem likely that these cases make as much as one fifth of the cases of gas infection. 
If moderate numbers occur, the evidence is of little more value than is obtained by culture alone.

The presence by culture alone reduces the chances from $1: 30$ against a given wound in this series being gas infected to $1: 7$, and hence is so slight evidence as to be hardly worth considering.

It should be emphasized that these figures apply only to wounds of the special character described. It seems probable that in wounds before excision or when excision is not carefully done, neither smear nor culture can afford very valuable evidence on account of the common occurrence of $B$. a'elchii.

\section{RELATION TO SUTURE}

Of nine cases with $B$. welchii on culture at time of suture, two were failures, developing gas infection, and one a part failure. Of the remainder, $B$. welchii persisted, with positive smears, harmlessly for various feriols after the suture; in two cases it persisted for three days; in one, for four days, and in two, from one to two days. One case of positive culture was oltained five days after suture. The two cases of failure were in wounds of from one to two days, and large numbers of $B$. zelchii were present in each case. In no successful case were more than very moderate numbers present at the time of suture. As far as the evidence of this small number of cases goes, it does not seem that the mere presence of $B$. welchii is a contraindication for suture, but that its presence in large numbers is. In none of the cases were streptococci present. The significance of the presence of $B$. zuelchii may be entirely different when associated with various organisms.

\section{SUMMARY AND CONCLUSIONS}

1. In a consecutive series of 137 wounds aged from one to eleven days, in patients arriving at a base hospital during a quiet period after thorough casualty clearing station treatment, $B$. z'elchii was obtained by culture in 23 per cent. Cases with more than extremely few $B$. welchii organisms on smear made 14 per cent. of the series. These figures are much lower than for wounds during rush periods, or for wounds before excision was common.

2. The small difference in the occurrence of $B$. wrolchii in the series in the excised, compared with the nonexcised, is due to good judgment in excising especially the wounds in which $B$. zuelchii is most apt to be present.

3. Definite muscle gas infection occurred in 2.9 per cent. of the series. Including slightly doubtful cases, the incidence is 5.1 per cent. Of the cases with $B$. avelchii on culture, the cases with unquestionable infection number 13 per cent., and including the slightly doubtful cases, 22.5 per cent.

4. For further studies, cases of gas infection were included from a previous nonconsecutive series. These cases were practically all in excised wounds. It was found that the cultures for $B$. avelchii tend to become negative by the eighth day when no gas infection has uccurred, but to last until the twelfth day or later in the cases with gas infection. Even reckoning the day of operation as the starting point, persistence is distinctly greater in the gas-infected wounds. This is in spite of complete lack of evidence of active infection after the thorough operation performed in each case.

5. There is a definite tendency to persist in greater numbers until the eighth day in the gas-infected cases than in those not infected.
6. In cases without gas infection, the numbers of bacilli tended to become very small early in the history of the wound.

7. The results suggest that $B$. welchii does not flourish long in surface exudate. Persistence in cases without gas infection is probably due largely to previously unrecognized local infection, or to small anaerobic foci in large wounds in which a thorough excision was impracticable. The persistence after gas infection may be explained by a gradual riddance of the bacilli from invaded but not definitely altered tissues.

8. In wounds of the character of this series, the presence of $B$. welchii in large numbers is good evidence of an active gas infection. The evidence is stronger, the older the wound. Its absence is strong but not conclusive evidence of lack of infection, for negative cultures are occasionally obtained when $B$. zuelchii is not a factor in the infection, or when the infection begins deep about a foreign body.

9. If moderate numbers of $B$. welchii are present, the evidence is of about the same value as is given by culture alone. The value of culture alone is so slight as scarcely to be worth considering.

These facts with regard to the value of smear and culture with reference to the diagnosis of gas infection in these wounds appear to rest on the inability of $B$. welchii to grow luxuriantly for a long period on the surface of surgically cleaned wounds, thus preventing the occurrence of large numbers when no infection is present; and on the occurrence of large numbers of $B$, welchii in the exuded serum of the infected cases, thus causing the highly positive smears.

10. The statements made here probably do not apply to wounds that have not had thorough previous surgical treatment.

11. The presence of $B$. welchii in large numbers was a contraindication to sutures in the small series observed. Its presence in small numbers is not necessarily a contraindication. There are points here in connection with the association of bacteria that need investigation. $B$. welchii may persist harmlessly as long as five days after suture.

"Team Work" by the Medical Profession.-In a summary of that portion of the Cavendish lecture by Sir Bertrand Dawson relating to the future of the medical profession, the Medical Officer quotes him as having said that the growth of knowledge makes measures for the prevention and cure of disease more and more complex, so that the full exercise of these powers no longer lies within the scope of individual effort but requires for its accomplishment well organized "team work." In the future, investigation will become more and more technical and collective treatment correspondingly specialized. This renders the patient's home less suited for his treatment and the need for institutional facilities all the greater. Sketching the scheme which he would consider best for fostering a closer union between the health organization of the community and the intellectual and professional life of the doctors, he said that the nucleus of this system of medical service would be the hospital. Local hospitals and clinics would be grouped in relation to central and teaching hospitals in the larger towns, and there would be corresponding relationship between the local practitioner and the consultant of the district. From this it would follow that the practitioner's source of income would be more in the nature of a salary than of fees, but this would not mean the extinction of private practice. A full-time salaried service for all medical men he regarded as detrimental to the best interests of both doctor and patient, for medicine stereotyped under such a system would become a "soulless machine." 